\section{Diarréia por rotavírus em crianças desnutridas hospitalizadas no Instituto Materno Infantil Prof. Fernando Figueira, IMIP}

\section{Rotavirus induced diarrhea in malnourished children hospitalized at the Instituto Materno Infantil Prof. Fernando Figueira, IMIP}

Renata Cavalcanti Cauás 1 Ana Rodrigues Falbo 2 Jailson de Barros Correia 3 Kaline Maria Maciel de Oliveira 4 Fernanda Maria Ulisses Montenegro 5

1-5 Grupo Saúde da Criança. Departamento de Pesquisa. Instituto Materno Infantil Prof. Fernando Figueira, IMIP. Rua dos Coelhos, 300. Recife, PE, Brasil. CEP: 50.070-550

\begin{abstract}
Objectives: to establish rotavirus incidence in diarrheic diseases in children from 0 to five years of age, with moderate and severe malnutrition hospitalized at Instituto Materno Infantil Prof. Fernando Figueira (IMIP).

Methods: a descriptive case study series was performed from August 2004 to June 2005. Fifty children comprised the study. A pre-codified questionnaire was used to build a data base on the Epi-info version 6.0 software. Stool samples were analyzed and the positive ones were selected by electronic microscopy for rotavirus and then tested by enzymatic immunoassay.

Results: the incidence of rotavirus in the studied group was of $24.0 \%$. The families were of low income, $96 \%$ had per capita income below $R \$ 150.00$. Near two thirds of the children (60.4\%) were breast feed for less than three months and 10\% of them had never been vaccinated.

Conclusions: the study contributes to the etiology of diarrheic disease in malnourished children, nevertheless larger series are required to validate the results.
\end{abstract}

Key words Diarrhea infantile, Rotavirus, Child Nutrition disorders

\section{Resumo}

Objetivos: verificar a freqüência do rotavírus em crianças de zero a cinco anos com doença diarréica e desnutrição moderada e grave, hospitalizadas no Instituto Materno Infantil Prof. Fernando Figueira (IMIP).

Métodos: foi realizado um estudo série de casos durante o período de agosto de 2004 e junho de 2005. No total fizeram parte do estudo 50 crianças. Utilizou-se um questionário pré-codificado e foi montado um banco de dados no software Epi-info 6.0. As amostras de fezes foram analisadas quanto à presença do rotavírus, através de técnicas de imunoensaio enzimático.

Resultados: verificou-se que a freqüência do rotavírus na doença diarréica do grupo estudado foi de 24,0\%. Em geral as famílias eram pobres, 96,0\% delas com renda familiar per capita inferior a $R \$ 150,00$. Com relação às crianças dois terços $(60,4 \%)$ receberam leite materno por um período inferior a três meses e $10 \%$ delas nunca haviam sido vascinadas.

Conclusões: o estudo traz contribuição para o conhecimento da etiologia da diarréia na criança com desnutrição, no entanto, se faz necessária a realização de estudos com um maior número de crianças para que os resultados possam ser mais conclusivos.

Palavras-chave Diarréia infantil, Rotavirus, Transtornos da nutrição infantil 


\section{Introdução}

A desnutrição na infância em termos populacionais é uma doença de origem multifatorial e complexa que tem suas raízes na pobreza. Ocorre quando o organismo não recebe os nutrientes necessários para o seu metabolismo fisiológico, devido à falta de aporte ou problema na utilização do que lhe é ofertado. Assim sendo, na maioria dos casos, a desnutrição é o resultado de uma ingestão insuficiente, ou fome, e de doenças. ${ }^{1}$

Das cerca de 12 milhões de mortes em menores de cinco anos registradas a cada ano nos países em desenvolvimento, cerca de sete milhões podem ser atribuídas direta ou indiretamente á desnutrição. ${ }^{2}$

No início do ano 2000, estimava-se que cerca de $30,0 \%$ da população mundial sofria de alguma forma de desnutrição, e em 2005, estima-se uma prevalência de desnutrição infantil de $29,0 \%$, o continente Africano apresentando a maior taxa com 33,8\%, seguido da Ásia com $29,9 \%$ e da América Latina com $9,3 \% .3$

No Brasil, das crianças na faixa etária inferior a cinco anos, em 2002, 7,0\% era afetadas por desnutrição crônica e 2,0\% por desnutrição aguda. 4 Em Pernambuco, segundo dados da II Pesquisa Estadual de Nutrição e Saúde (PENS) realizada em 1997, $12,1 \%$ de crianças menores de cinco anos apresentavam desnutrição crônica e 1,8\% desnutrição aguda. 5

A desnutrição afeta todos os sistemas e órgãos, nenhuma das funções até o momento estudadas nesta condição tem se mostrado normal. De longe, a adaptação mais importante é a redução do trabalho corporal em todos os seus níveis de organização. 6

Quanto ao trato gastroentestinal na desnutrição, há uma tendência à diminuição do tamanho do enterócito, do seu núcleo e do bordo em escova, à medida que aumenta o grau de desnutrição. ${ }^{7}$

A secreção ácida gástrica está reduzida, permitindo a colonização do estômago, que é normalmente estéril, por bactérias intestinais fecais. As bactérias competem tanto pela vitamina B-12, como levam a uma consequiência mais grave que é a desconjugação dos sais biliares, com a interferência na absorção das gorduras. A cinética da regeneração do epitélio intestinal está alterada e há lentificação na proliferação, migração e maturação das células. A conseqüência é a redução das enzimas dissacaridases, especialmente a lactase. 8

Além dos mecanismos citados anteriormente serem fatores predisponentes para a presença de diarréia na criança desnutrida, a desnutrição tem efeitos adversos no mecanismo imunológico especí- fico e inespecífico, aumentando a susceptibilidade às infecções (diarréia e pneumonia), estabelecendo um ciclo vicioso, com agravamento do estado nutricional por diminuição da ingestão, aumento das perdas, má absorção e comprometimento da mobilização dos estoques corporais. ${ }^{9-12}$

O Rotavírus é um dos mais importantes agentes etiológicos da doença diarréica na criança de modo geral. Está associado a 30,0-50,0\% dos casos de diarréia grave e aproximadamente com um terço das hospitalizações por diarréia, contribuindo com 800.000 mortes em todo o mundo. 13

No Brasil, denota-se a maior expressão clínica dos episódios de diarréia relacionados aos rotavírus se comparados àqueles por outros patógenos, tanto na comunidade como no âmbito hospitalar. 14 Nos hospitais os parâmetros clínicos como febre, vômitos e desidratação prevaleceram amplamente nas diarréias associadas aos rotavírus, com base em análise recente. 15

Quanto à fisiopatologia o rotavírus age penetrando nos enterócitos, os quais se rompem e são substituídos por células absortivas imaturas. Essas se caracterizam por apresentar números reduzidos de microvilosidades, embora conservem sua propriedade secretória. Observa-se redução da $\mathrm{Na} / \mathrm{K}$ ATPase, responsável pelo processo de absorção intestinal do $\mathrm{Na}$ acoplado á glicose, havendo redução da absorção deste assim como da água. Os níveis de AMP cíclico se mantêm inalterados, daí excluir-se o mecanismo secretório no contexto fisiopatológico das diarréias por rotavírus. Há também redução da atividade inerente ás dissacaridases, principalmente da lactase, comprometendo o desdobramento dos dissacarídeos e sua absorção, promovendo um aumento da osmolaridade do lúmen intestinal, e conseqüentemente, aumento do afluxo de líquido. $\mathrm{O}$ açúcar não absorvido pode sofrer ação de bactérias que colonizam as porções mais distais do intestino, resultando na eliminação de fezes com pH ácido, resultando na diarréia de natureza osmótica. 16

A identificaçãoda participação do rotavírus na etiologia da diarréia na região Nordeste, não só preenche uma importante lacuna no conhecimento, pois não há dados sobre a prevalência da infecção pelo rotavírus, especialmente no grupo de crianças desnutridas graves, - como também trará grande contribuição para adequar o manejo clínico dos casos, diminuindo a morbimortalidade pela doença diarréica.

O objetivo do estudo foi verificar a prevalência do rotavírus na doença diarréica de crianças com desnutrição moderada e grave hospitalizadas no Instituto Materno Infantil de Pernambuco (IMIP). 


\section{Métodos}

Foi realizado um estudo série de casos, do qual participaram inicialmente todas as 54 crianças de zero a 60 meses, com desnutrição moderada e grave, caracterizadas pelo índice peso/altura menor que 2DP, utilizando o padrão de referência do National Center Health Statistics (NCHS) e da Organização Mundial de Saúde (OMS), e/ou presença de edema simétrico envolvendo no mínimo os pés, hospitalizadas no IMIP, durante o período entre agosto de 2004 e junho de 2005. Para as crianças com comprimento menor que $49 \mathrm{~cm}$ sem padronização para utilização do indicador peso/altura foi utilizado o critério clínico de emagrecimento extremo. Quatro crianças foram excluídas, pois houve perda das amostras fecais antes do envio para a realização da pesquisa do rotavírus. No total fizeram parte do estudo 50 crianças.

Para coleta dos dados foi utilizado um formulário pré-codificado, aplicado ao responsável pela criança, elaborado conforme as seguintes variáveis do estudo: freqüência do rotavírus; variáveis da condição sociodemográfica (renda familiar per capita, local de moradia, saneamento da moradia); características da mãe (idade, escolaridade, situação de emprego); variáveis da condição biológica e clínica no momento da admissão (idade, sexo, prematuridade, aleitamento materno, estado vacinal, febre, vômito, desidratação, tipo de diarréia, duração do episódio diarréico no momento da admissão) e do manejo durante o internamento (uso de antibioticoterapia e evolução para alta ou óbito).

Amostras de fezes recém-emitidas foram colhidas (através de saco coletor ou com auxílio de uma espátula a partir das fraldas das crianças participantes no estudo) e acondicionadas em reservatórios de plástico impermeáveis devidamente identificados e armazenados sob congelamento a $-20^{\circ}$ graus Celsius. As amostras foram transportadas, via aérea, de acordo com as normas técnicas nacionais e internacionais da Agência Nacional de Vigilância Sanitária (ANVISA) para o transporte de material biológico, para serem analisadas em laboratório da Universidade de Liverpool, Inglaterra.

As amostras fecais foram triadas para a presença do rotavírus por microscopia eletrônica. As amostras foram suspensas a $10 \%$ em solução salina tamponada em fosfato (PBS, pH 7,2) e colocadas em placas de cobre pré-fixadas com resina. As placas foram negativamente coradas em ácido fosfotúngstico a $2 \%$ e visualizadas em microscópio eletrônico (Philips 301), com aumento inicial de 45.000 vezes.
As amostras positivas à microscopia eletrônica foram então testadas para a presença de rotavírus, através de técnicas de imunoensaio enzimático.

A partir do levantamento das informações nos formulários pré-codificados, foi construído um banco de dados (com dupla entrada), no software Epi-info versão 6.0. Para análise do estado nutricional, foi utilizado o Epi-nut, módulo do Epi-info, com o padrão do NCHS.

\section{Resultados}

Verificou-se que a prevalência do rotavírus na doença diarréica do grupo estudado foi de $24,0 \%$ como mostrado na Tabela 1 .

Em relação às características sociodemográficas estudadas, foi observado que em geral as famílias eram pobres sendo $96,0 \%$ delas com renda familiar per capita inferior a $\mathrm{R} \$ 150,00$ e $52,0 \%$ residindo no interior do Estado. Esses e outros dados referentes à condição da moradia encontram-se na Tabela 2.

A maior parte das mães das crianças tinha idade entre 19 e 30 anos, 70,0\% delas tinham menos de oito anos de escolaridade e 78,0\%, trabalhavam em casa (Tabela 3).

$\mathrm{Na}$ Tabela 4 encontram-se algumas características da condição biológica e clínica das crianças: $56 \%$ eram maiores de um ano de idade, $16,0 \%$ das crianças foram prematuras ao nascimento e aproximadamente dois terços $(60,4 \%)$ mamou por um período inferior a três meses. $10,0 \%$ delas nunca haviam sido vacinadas.

Segundo anamnese e exame clínico realizado no momento da admissão hospitalar, para o grupo como um todo, 50,0\% apresentavam desidratação no exame físico inicial. A presença de febre e vômito segundo história clínica realizada na admissão foi de $74,0 \%$ e $76,0 \%$ respectivamente. Quanto ao tipo de

\section{Tabela 1}

Distribuição de freqüência das crianças com desnutrição moderada e grave e com diarréia, hospitalizadas no Instituto Materno Infantil Prof. Fernando Figueira IMIP, segundo a presença do rotavírus nas fezes. Recife, Pernambuco, 2004 a 2005.

\begin{tabular}{lcc}
\hline Rotavírus & N (50) & $\%$ \\
\hline Sim & 12 & 24,0 \\
Não & 38 & 76,0 \\
\hline
\end{tabular}


Tabela 2

Distribuição de freqüência das crianças com desnutrição moderada e grave e com diarréia, hospitalizadas no Instituto Materno Infantil Prof. Fernando Figueira IMIP, segundo características da sua condição sóciodemográfica. Recife, Pernambuco, 2004 a 2005.

\begin{tabular}{lrr}
\hline Variaveis & N (50) & $\%$ \\
\hline Renda per capita mensal & & \\
$\quad<$ R $\$ 150,00$ & 48 & 96,0 \\
$\quad$ R $\$ 150,00$ & 2 & 4,0 \\
Local de residência & & \\
$\quad$ Região Metropolitana do Recife & 24 & 48,0 \\
$\quad$ Zona da Mata & 20 & 40,0 \\
$\quad$ Agreste & 2 & 4,0 \\
$\quad$ Sertão & 3 & 6,0 \\
$\quad$ Vale do São Francisco & 1 & 2,0 \\
Água encanada & & \\
$\quad$ Sim & 37 & 74,0 \\
Não & 13 & 26,0 \\
Fossa ou esgoto & & \\
$\quad$ Sim & 35 & 70,0 \\
Não & 15 & 30,0 \\
Recolhimento de lixo & & \\
$\quad$ Sim & 38 & 76,0 \\
Não & 12 & 24,0 \\
\hline
\end{tabular}

Tabela 3

Distribuição de freqüência das crianças com desnutrição moderada e grave e com diarréia, hospitalizadas no no Instituto Materno Infantil Prof. Fernando Figueira IMIP, segundo características das mães. Recife, Pernambuco, 2004 a 2005.

\begin{tabular}{lrr}
\hline Variaveis & N (50) & $\%$ \\
\hline Idade em anos & & \\
$\quad<19$ & 5 & 10,0 \\
19 a 30 & 36 & 72,0 \\
$\quad \geq 30$ & 9 & 18,0 \\
Escolaridade $(\mathrm{n}=49)^{*}$ & & \\
$\quad$ Nenhuma & 5 & 10,2 \\
$\quad<8$ anos & 40 & 81,6 \\
$\quad \geq 8$ anos & 4 & 8,2 \\
Ocupação & & \\
$\quad$ Trabalha no lar & 39 & 78,0 \\
$\quad$ Trabalha fora de casa & 11 & 22,0 \\
\hline
\end{tabular}

*A não correspondência com o total da amostra se deve à ocorrência de mais de um sintoma em algumas crianças

\section{Tabela 4}

Distribuição de freqüência das crianças com desnutrição moderada e grave e com diarréia, hospitalizadas no Instituto Materno Infantil Prof. Fernando Figueira IMIP, segundo características da sua condição biológica e clínica. Recife, Pernambuco, 2004 a 2005.

\begin{tabular}{|c|c|c|}
\hline Variaveis & $N(50)$ & $\%$ \\
\hline \multicolumn{3}{|l|}{ Idade (meses) } \\
\hline$<6$ & 16 & 32,0 \\
\hline 6 a 12 & 6 & 12,0 \\
\hline$\geq 12$ & 28 & 56,0 \\
\hline \multicolumn{3}{|l|}{ Sexo } \\
\hline Masculino & 29 & 58,0 \\
\hline Feminino & 21 & 42,0 \\
\hline \multicolumn{3}{|l|}{ Prematuridade* } \\
\hline Sim & 8 & 16,0 \\
\hline Não & 41 & 82,0 \\
\hline \multicolumn{3}{|c|}{ Aleitamento materno $(n=43)^{*}$} \\
\hline Nunca mamou & 6 & 13,9 \\
\hline$<3$ meses & 26 & 60,5 \\
\hline 3 a 6 meses & 4 & 9,3 \\
\hline$\geq 6$ meses & 7 & 16,3 \\
\hline \multicolumn{3}{|l|}{ Estado vacinal $(n=43)^{*}$} \\
\hline Nenhuma vacina & 5 & 10,0 \\
\hline Esquema incompleto & 14 & 28,0 \\
\hline Esquema completo & 24 & 48,0 \\
\hline
\end{tabular}

*A não correspondência com o total da amostra se deve a falta da informação em relação às variáveis pesquisadas

Tabela 5

Distribuição de freqüência das crianças com desnutrição moderada e grave e com diarréia, hospitalizadas no Instituto Materno Infantil Prof. Fernando Figueira IMIP, segundo características de sua anamnese e exame clínico realizado no momento da admissão hospitalar. Recife, Pernambuco, 2004 a 2005.

\begin{tabular}{lcc}
\hline Variaveis & N (50) & $\%$ \\
\hline Desidratação & 25 & 50,0 \\
Febre & 37 & 74,0 \\
Vômito & 38 & 76,0 \\
Tipo de diarréia & & \\
$\quad$ Aquosa & 44 & 88,0 \\
$\quad$ Disenteria & 6 & 12,0 \\
Duração da diarréia & & \\
$\quad<14$ dias (aguda) & & \\
$\quad \geq 14$ dias (persistente) & 40 & 80,0 \\
& 10 & 20,0 \\
\hline
\end{tabular}


Tabela 6

Distribuição de freqüência das crianças com desnutrição moderada e grave e com diarréia por rotavírus, segundo aspectos clínicos na admissão e segundo evolução para alta ou óbito. Recife, Pernambuco, 2004 a 2005.

\begin{tabular}{lrc}
\hline Variaveis & N (12) & $\%$ \\
\hline Desidratação à admissão & 7 & 58,3 \\
Febre & 7 & 58,3 \\
Vômitos & 11 & 91,8 \\
$\quad$ Tipo da diarréia & & \\
$\quad$ Aquosa & 12 & 100,0 \\
$\quad \begin{array}{l}\text { Duração da diarréia } \\
\quad \text { Aguda }\end{array}$ & & \\
$\quad$ Persistente & 10 & 83,3 \\
$\quad$ Evolução & 12 & 16,7 \\
$\quad$ Alta hospitalar & & \\
\hline
\end{tabular}

diarréia constatou-se que $88,0 \%$ apresentavam diarréia aquosa e $12,0 \%$ disenteria. Oitenta por cento das crianças apresentavam o quadro de diarréia por menos de 14 dias (diarréia aguda) até o momento do internamento e $20,0 \%$ delas por mais de 14 dias (diarréia persistente) (Tabela 5).

Quanto às 12 crianças com doença diarréica causada por rotavírus, sete apresentaram algum grau de desidratação no momento da admissão hospitalar, sete delas estavam com febre e onze com vômitos no mesmo período. Em todas as 12 crianças a diarréia foi do tipo aquoso e quanto à duração do episódio diarréico, dez apresentaram diarréia aguda e duas diarréia persistente. Todas receberam alta hospitalar (Tabela 6).

Quanto ao manejo das crianças durante o internamento, constatou-se que $90,0 \%$ delas fizeram uso de antibioticoterapia. Constatou-se que das 50 crianças do estudo, $80,0 \%$ receberam alta hospitalar e $20,0 \%$ foram a óbito.

\section{Discussão}

Encontrou-se o rotavírus nas fezes diarréicas de $24,0 \%$ das crianças estudadas. Esse resultado é semelhante àqueles encontrados na literatura pesquisada, cujos resultados apresentam uma freqüência variando de $5,0 \%$ a $71,0 \%$. No entanto, nesses estudos não se levou em conta a situação nutricional das crianças. Estudo realizado na Índia encontrou uma freqüência da diarréia por rotavírus variando entre 5,0-71,0\% em crianças menores de cinco anos, hospitalizadas. 13

$\mathrm{Na}$ Turquia, em estudo realizado para determinar a freqüência e significância clínica da gastroenterite pelo rotavírus, em crianças menores de cinco anos, o rotavírus foi identificado em $39,8 \%$ das crianças, sendo $80,7 \%$ das mesmas com idade inferior a dois anos. Verificou-se também que $69,0 \%$ das crianças com infecção pelo rotavírus apresentavam gastroenterite grave. 17

A maioria das investigações conduzidas na América latina, em crianças abaixo de cinco anos de idade, em nível hospitalar ou ambulatorial, aponta para uma ampla variação nas taxas de freqüência, podendo-se citar: Argentina 12,9 a 34\%; Brasil 13 a $40 \%$; Chile 11,4 a $40 \%$; Costa Rica 45,3 a $60 \%$ e Venezuela 30 a $50 \% .18$

No Brasil, vários estudos foram realizados, abrangendo as diferentes regiões do país, com base nos quais se determinaram múltiplas taxas relativas à freqüência das diarréias por rotavírus entre crianças com idades inferiores a seis anos. O contingente sob investigação era representado em sua quase totalidade por demanda ambulatorial ou hospitalar. Em termos gerais, a freqüência de diarréias associadas à rotavírus variou de 12,0\% a 42,0\%.19

Há poucos estudos publicados sobre etiologia da diarréia em crianças com desnutrição. Segundo Kakai et al.,20 em estudo realizado em 1995, o isolamento de Campylobacter em fezes diarréicas é maior em crianças desnutridas quando comparado às de boa situação nutricional. Fagundes - Neto e Andrade, 21 em 1999, em estudo sobre risco de letalidade em crianças hospitalizadas com diarréia e desnutrição verificaram que a E.coli (EPEC) foi o enteropatógeno mais freqüentemente isolado.

Segundo Mpabalwani et al.22 a infecção por rotavírus é mais comum em crianças com bom estado nutricional do que naquelas com desnutrição. Isto condiz então com o resultado encontrado no presente estudo, no qual a maioria $(76,0 \%)$ das crianças possuía outra etiologia para a diarréia que não o rotavírus. Já segundo Dagan et al. ${ }^{23}$ o rotavírus é encontrado em iguais proporções tanto em crianças com bom estado nutricional quanto naquelas desnutridas.

Oliveira et al. ${ }^{24}$ observaram que as crianças com diarréia por rotavírus apresentam início abrupto com vômitos em mais da metade dos casos seguidos de febre alta e diarréia profusa. Estudo realizado por Linhares 19 evidenciou a gravidade da diarréia por rotavírus, quando comparada com a de outros enteropatógenos, ao registrar uma maior frequiência de febre $(65,9 \%)$ e do número de evacuações no 
grupo relacionado aos rotavírus. No presente estudo, as 12 crianças nas quais se isolou o rotavírus nas fezes apresentaram quadro clínico semelhante ao descrito pelos autores citados acima.

Quanto às características da população estudada e considerando os dois eventos, diarréia e desnutrição, há compatibilidade com famílias pobres, com baixa renda familiar per capita mensal, precárias condições da moradia e que em se tratando de uma população hospitalar e, portanto, de casos mais graves. Igualmente crianças com alguns fatores de risco como idade abaixo de 12 meses e prematuridade, bem como desmame precoce, situação vacinal incompleta e mães com baixa escolaridade.

Segundo trabalho publicado por Parashar et al.,13 em 2003, a doença diarréica causada por rotavírus tem freqüência similar em países desenvolvidos e naqueles em desenvolvimento. No entanto nos países em desenvolvimento tendem a morrer mais devido a vários fatores, dentre eles possivelmente, o pequeno acesso à terapia de rehidratação oral e à elevada prevalência da desnutrição infantil.

Considerando os resultados apresentados, observa-se que a presença do rotavírus nas fezes diarréicas das crianças desnutridas é importante. A

\section{Referências}

1. WHO (World Health Organization), ACC (Administrative Commitee on Coordination), SCN (Standing Commitee on Nutrition). Nutrition throught life: $4^{\text {th }}$ report on the World Nutrition Situation. Geneva; 2000.

2. Bryce J, el Arifeen S, Pariyo G, Lanata C, Gwatkin D, Habicht JP. Reducing child mortality: can public health deliver? Lancet. 2003; 362: 159-64.

3. Onis M, Frongillo EA, Blüssner M. Is malnutrition declining? An analysis of change in levels of child malnutrition since 1980. Bull World Health Organ. 2000; 78: 1222-3.

4. UNICEF (United Nations International Children's Emergency Fund). Panorama: Brasil - statistics [on line]. Available: http: //www.unicef.org/spanish/infobycountry/ brazil-statistics.html>. (2004 Sep 1).

5. Batista Filho M, Romani SAM. Alimentação, nutrição e saúde no Estado de Pernambuco: espacialização e fatores socioeconômicos. Recife: IMIP; 2002. (Série de Publicações Científicas do Instituto Materno Infantil Prof. Fernando Figueira, IMIP, n. 7).

6. Ashworth A. Emergency balance and growth: experience in treating children with malnutrition. Kidney Int. 1978, 14: 301-5.

7. Pires ALG, Pires TRS, Silva VD. Estudo morfométrico e estereológico digital da mucosa do intestino delgado de crianças eutróficas e desnutridas com diarréia persistente. J Pediatr. (Rio J) 2003; 79: 329-36. própria desnutrição é um importante fator de risco para a gravidade da doença diarréica por rotavírus, no entanto, com pouca associação com um desfecho fatal. 24 Esse fato foi confirmado pelo presente estudo, uma vez que todas as crianças com diarréia causada por rotavírus tiveram alta hospitalar.

É importante definir o papel do rotavírus na etiologia da diarréia na criança de modo geral, e especialmente na criança desnutrida. De fato uma vez que se confirme a sua elevada ocorrência, podese ter mais segurança na redução do uso da antibioticoterapia, diminuindo assim os custos, os efeitos colaterais indesejados dessas drogas, principalmente no caso da doença diarréica, adequando dessa forma o seu manejo e contribuindo para a redução da morbimortalidade infantil.

\section{Agradecimentos}

Ao Instituto Materno Infantil Prof. Fernando Figueira, IMIP pelo suporte e ao Conselho Nacional de Desenvolvimento Científico e Tecnológico, CNPQ pela bolsa do Programa de Iniciação Científica.
8. Solomons NW. Rehabilitating the severely malnourished infant and child. J Am Diet Assoc. 1985; 85: 28-39.

9. Brewster DR, Manary MJ, Menzies IS, O'Loughlin EV, Henry RL. Intestinal permeabilitity in kwashiorkor. Arc Dis Child. 1997; 76: 236-41.

10. Cunha AL. Relationship between acute respiratory infection and malnutrition in children under five years of age. Acta Paediatr. 2000; 89: 608-9.

11. Giovambattista A, Spinedi E, Sanjurjo A, et al Circulating and mitogen-induced tumor necrosis factor (TNF) in malnourished children. Medicina. (Buenos Aires) 2000; 60: 339-42.

12. Kossmann J, Nestel P, Herrera MG, Amim EI, Fawzi WW. Undernutrition in relation to childhood infections: a prospective study in the Sudan. Eur J Clin Nutr. 2000; 54: 463-72.

13. Parashar UD, Hummelman EG, Breese JS, Miller AM, Glass RI. Global illness and deaths caused by rotavirus disease in children. Emerg Infect Dis. 2003; 9: 565-72.

14. Linhares AC, Gabbay YB, Freitas ES, da Rosa EST, Mascarenhas JDP, Loureiro ECB. Longitudinal study of rotavirus among children from Belém, Brazil. Epidemiol Infect. 1989; 102: 129-45.

15. Gusmão RHP, Mascarenhas JDP, Gabbay YB, Lins-Lainson Z, Ramos FLP, Monteiro TAF, Valente SA, Linhares AC, Fagundes-Neto U. Rotaviruses as a cause of nosocomial, infantile diarrhoea in Northern Brazil: pilot study. Mem Inst Oswaldo Cruz. 1995; 90: 743-9. 
16. Pereira HG, Linhares AC, Candeias JAN, Glass RI. National laboratory surveillance of viral agent of gastroenteritis in Brazil. Bull Pan American Health Org. 1994; 27 : 224-33.

17. Kurugöl Z, Geylani S, Karaca Y, Umay F, Erensoy S, Vardar F, Bak M, Yaprak I, Özkinay F, Özkinay C. Rotavirus gastroenteritis among children under five years of age in Izmir, Turkey.Turk J Pediatr. 2003; 45: 290-4.

18. Linhares AC, Breese JS. Rotavirus vaccines and vaccination in Latin America. Pan Am J Public Health. 2000; 8: 305-31.

19. Linhares AC. Epidemiologia das infecções por rotavírus no Brasil e os desafios para o seu controle. Cad Saúde Pública. 2000; 16: 629-46

20. Kakai R, Wamola IA, Bwayo JJ, Ndinya-Achola JO. Enteric pathogens in malnourished children with diarrhea. East Afr Med J. 1995; 72: 288-9.
21. Fagundes-Neto U, de Andrade JA. Acute diarrhea and malnutrition: lethality risk in hospitalized infants. J Am Coll Nutr. 1999; 18: 303-8.

22. Mpabalwani M, Oshitani H, Kasolo F, Mizuta K, Luo N, Matsubayashi N, Bhat G, Suzuki H, Numazaki Y. Rotavirus gastro-enteritis in hospitalized children with acute diarrhoea in Zâmbia. Ann Trop Paediatr. 1995; 15: 39-43.

23. Dagan R, Bar-David Y, Sarov B, Katz M, Kassis I, Greenberg D, Glass RI, Margolis CZ, Sarov I. Rotavirus diarrhea in Jewish and Bedouin children in the Negev region of Israel: epidemiology, clinical aspects and possible role of malnutrition in severity of illness. Pediatr Infect Dis J. 1990; 9: 314-21.

24. Oliveira CS, Gabbay YB, Linhares AC. Gastroenterites virais. In: Farhat CK, Carvalho ES, Carvalho LHFR, Succi RCM. Infectologia pediátrica. 2. ed. São Paulo: Atheneu; 1999

Recebido em 30 de outubro de 2005

Versão final apresentada em 28 de dezembro de 2005

Aprovado em 19 de janeiro de 2006 